zustand blieben in dieser Zeit von Vorhofflimmern im Sinne des primären Endpunktes verschont.

Darüber hinaus testeten die Autoren den Nutzen eines Trainings zur Verbesserung der Fitness im Laufe der Studie. Die Patienten trainierten dazu regelmäßig drei bis fünf strukturierte Einheiten aeroben Ausdauer- oder Krafttrainings niedriger bis mäßiggradiger Intensität mit einer Wochen-Trainingszeit von 60200 Minuten. In der Auswertung unterschieden sie Patienten, die zu Studienende ihre Ausdauer um mehr als 2 MET („metabolic equivalent of task“) verbesserten von Patienten, die ihre Ausdauer um weniger als 2 MET verbesserten. $\mathrm{Pa}$ tienten, die ihrer Fitness um mehr als 2 MET verbesserten, verloren mehr $\mathrm{Ge}$ wicht (12 vs. 3 Kilogramm) und senkten ihren Blutdruck in stärkerem Ausmaß (systolisch -14 vs. $-10 \mathrm{mmHg}$ ).

Darüber hinaus sank die Zahl der benötigten Antihypertensiva deutlich. Auch Lipid- und Glukosestoffwechselparameter besserten sich signifikant, fer- ner auch das linksatriale Volumen und die linksventrikuläre diastolische Funktion als Ausdruck eines strukturellen kardialen Remodellings.

\section{Je fitter desto \\ geringer das Rezidivrisiko}

Hauptstudienendpunkt war indes die Freiheit von Vorhofflimmern im 7-TageEKG sowie die entsprechende Beschwerdefreiheit, und dies war bei $61 \%$ der $\mathrm{Pa}$ tienten mit Fitnesszuwachs über 2 MET der Fall, aber nur bei 18\% der Patienten mit geringerem Trainingserfolg.

Die Patienten mit mehr als 2 MET Fitnesszuwachs gaben weniger kardiale $\mathrm{Be}$ schwerden und insgesamt eine bessere Lebensqualität an. Pro 1 MET Fitnessgewinn reduzierte sich das Risiko für das Wiederauftreten der Rhythmusstörung um 12\%, berichtete Pathak.

Besonders gut schnitten Patienten ab, die ihre Fitness um über 2 MET erhöhten und gleichzeitig ihr Gewicht um 10\% reduzierten. Sie blieben zu $76 \%$ von Vorhofflimmern verschont, im Vergleich zu
44\% bei Patienten mit Gewichtsverlust von $10 \%$, aber ohne Fitness-Gewinn. Patienten ohne 10\%igen Gewichtsverlust erhöhten ihre Aussichten auf Rezidivfreiheit von 13 auf $37 \%$, wenn sie ihre Fitness steigerten.

\section{Fazit: Am besten schlank und fit}

Fazit: Sowohl die kardiorespiratorische Fitness zu Studienbeginn, der FitnessZugewinn während der Studie als auch eine erfolgreiche Gewichtsreduktion erwiesen sich alle drei als unabhängige Prädiktoren für das Wiederauftreten von Vorhofflimmern.

Entsprechend sind die Aussichten auf Arrhythmie-Freiheit am größten, wenn der übergewichtige VF-Patient sowohl abnimmt als auch regelmäßig körperlich trainiert.

Dr. med. Dirk Einecke

Quelle: EHRA Europace Cardiostim, Mailand; Pathak, RK, et al. Impact of CARDIOrespirator FITness on Arrhythmia Recurrence in Obese Individuals with Atrial Fibrillation: The CARDIO-FIT Study. JACC 2015; doi: 10.1016/j.jacc.2015.06.488

\title{
Lohnt die Antikoagulation bei atrialen Tachyarrhythmien?
}

\section{Oft wird bei Schlaganfallpatienten erst nachträglich ein zuvor unbe- kanntes Vorhofflimmern festgestellt. Hätte sich diese schwere Komplikation womöglich durch eine frühzeitige Antikoagulation verhindern lassen?}

Eiver ne Patientengruppe, die möglicherweise für Vorhofflimmern prädestiniert ist und Studiendaten zufolge ein erhöhtes Schlaganfallrisiko aufweist, sind Patienten mit atrialen Tachyarrhythmien oder ,atrial high rate episodes" (AHRE). Ob diese durch eine frühzeitige Antikoagulation vor Schlaganfall, systemischen Embolien oder kardiovaskulären Todesfällen geschützt werden können, wird nun in der NOAH-AFNET 6-Studie untersucht, einem Gemeinschaftsprojekt des deutschen Kompetenznetzes Vorhofflimmern und der „European Society of Cardiology“.

Wie Prof. Paulus Kirchhof von der Universität Birmingham beim EHRA Eurospace-Meeting 2015 berichtete, werden in die Studie über 65-jährige $\mathrm{Pa}$ - tienten mit einem CHA2DS2-VAScScore von mindestens zwei und dokumentierten Episoden atrialer Tachykardien aufgenommen, aber ohne Vorhofflimmern. Die Patienten werden doppelblind mit dem oralen Antikoagulans Edoxaban oder Placebo behandelt. In der Kontrollgruppe wird zudem der Wert von ASS getestet.

\section{EU-Zulassung für Edoxaban}

Edoxaban hat am 25.06.2015 die EU-Zulassung für die Schlaganfallprävention bei Vorhofflimmern sowie die Behandlung und Prävention von rezidivierenden tiefen Beinvenenthrombosen und Lungenembolien erhalten.

Der Zulassung zugrunde liegen die Daten der großen Studien ENGAGE AF-
TIMI 48 sowie HOKUSAI-VTE. Die Einführung von Edoxaban als LIXIA$\mathrm{NA}^{\circledast}$ wird früh im 2. Halbjahr 2015 erwartet.

Dr. med. Dirk Einecke

Quelle: Satelliten-Symposium „Atrial high rate episodes and stroke prevention", EHRA, Europace Cardiostim, Mailand, 22.06.2015 (Veranstalter: Daiichi-Sankyo)

\section{॥ ONLINE-TIPP}

\section{Kardiologie.org}

Mehr Beiträge vom EHRA EuropaceCardiostim in Mailand finden Sie online im Kongressdossier:

www.kardiologie.org/kardiologie/ ehra-europace-cardiostim-2015kongress/377298

Z. B. über Themen wie HeparinBridging bei Device-Implantation und Ablation, Biomarker zur Bestimmung des plötzlichen Herztodrisikos oder NOAKs bei Ablation von Vorhofflimmern. 